\title{
Trauma induced tissue survival in vitro with a muscle-biomaterial based osteogenic organoid system: a proof of concept study
}

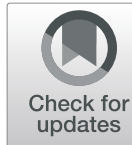

Tao He ${ }^{1,2^{*}}$, Jörg Hausdorf ${ }^{1}$, Yan Chevalier ${ }^{1}$ and Roland M. Klar ${ }^{1 *}$

\begin{abstract}
Background: The translation from animal research into the clinical environment remains problematic, as animal systems do not adequately replicate the human in vivo environment. Bioreactors have emerged as a good alternative that can reproduce part of the human in vivo processes at an in vitro level. However, in vitro bone formation platforms primarily utilize stem cells only, with tissue based in vitro systems remaining poorly investigated. As such, the present pilot study explored the tissue behavior and cell survival capability within a new in vitro skeletal muscle tissue-based biomaterial organoid bioreactor system to maximize future bone tissue engineering prospects.

Results: Three dimensional printed $\beta$-tricalcium phosphate/hydroxyapatite devices were either wrapped in a sheet of rat muscle tissue or first implanted in a heterotopic muscle pouch that was then excised and cultured in vitro for up to 30 days. Devices wrapped in muscle tissue showed cell death by day 15. Contrarily, devices in muscle pouches showed angiogenic and limited osteogenic gene expression tendencies with consistent TGF- $\beta_{1}, C O L 4 A 1$, VEGF-A, RUNX-2, and BMP-2 up-regulation, respectively. Histologically, muscle tissue degradation and fibrin release was seen being absorbed by devices acting possibly as a support for new tissue formation in the bioceramic scaffold that supports progenitor stem cell osteogenic differentiation.

Conclusions: These results therefore demonstrate that the skeletal muscle pouch-based biomaterial culturing system can support tissue survival over a prolonged culture period and represents a novel organoid tissue model that with further adjustments could generate bone tissue for direct clinical transplantations.
\end{abstract}

Keywords: Heterotopic implant model, In vitro, Tissue survival, Angiogenesis, Vasculogenesis, 3D printed $\beta-T C P / H A$, Pilot study, Organoid

\section{Background}

The effective translation from in vitro to in vivo and in vivo to clinical practice remains a major challenge for tissue regenerative sciences [1-3]. Whilst experimental in vitro and in vivo investigations continue to contribute greatly to deciphering specific criteria in biological sciences, the translation from a functional model to the clinical setting takes an exuberant amount of time and consumes vast resources [4]. This is one of the reasons why bone tissue induction models are not yet used and

\footnotetext{
*Correspondence: hetao@renji.com; roland.klar@med.uni-muenchen.de ${ }^{1}$ Department of Orthopedics, Physical Medicine and Rehabilitation, University Hospital of Munich (LMU), Munich, Germany

Full list of author information is available at the end of the article
}

the autogenous bone graft [5-9] remains the golden standard for bone regeneration clinically.

There is a clear need to develop more reliable ex vivo models where bioreactor platforms, simulating certain tissue types, have shown great capabilities at replicating certain in vivo environments $[10,11]$. However, bioreactors remain problematic for use in forming a complex structure like bone, as there are various biochemical, cellular and mechanical requirements that need to be met to form this tissue type either ectopically or orthotopically [12-19], where vascularization and/or angiogenesis are essential components that help the tissue survive and grow $[20,21]$.

Most bioreactor platforms utilize stem cells on a specific biomaterial to produce a specific single cell derived

(c) The Author(s). 2020 Open Access This article is distributed under the terms of the Creative Commons Attribution 4.0 International License (http://creativecommons.org/licenses/by/4.0/), which permits unrestricted use, distribution, and 
tissue type [22-24], which is inadequate for bone tissue morphogenesis as various steps are required that together culminate in the formation of this tissue [25]. Additionally, cells cultured in vitro not only lose their homeostatic state through the loss of essential amino acids, that growth medium can hardly supply in a controlled and released state as in vivo tissue breakdown would [26], but also need to develop a viable extracellular matrix (ECM) environment first before they can thrive and grow [27, 28]. Hence, in vivo tissue based bone inductive studies remain to date the best models to study the effect of biomaterial behavior in vivo. As such, a tissue-based bioreactor platform [23, 29,30 ] could be superior to that of a stem cell-based system as tissues possesses various biochemical building blocks and adult stem cell niches together with preestablished cell growth promoting environments that theoretically could provide a superior culturing milieu. However, the use of bone directly as a biomaterial growth environment in vitro is highly problematic, as culture medium cannot adequately diffuse across a hard tissue barrier [30]. Therefore, the present pilot study investigated the feasibility of two in vitro skeletal muscle-based biomaterial-culturing systems as this tissue, being considered among the promising candidate grafts for bone tissue engineering, allows for better nutrient flow [31-33]. The primary objective was to see if the models would support tissue survivability and growth into a custom threedimensional (3D) printed bone inductive biomaterial [34, 35], whereas the secondary objective was to determine if any vasculo-/angiogenic morphogenesis, by monitoring transcriptional and translational markers, would take place as this is a crucial component required for nascent bone tissue formation $[20,21]$.

\section{Results}

Tissue pouch model supported superior tissue survival and transformation than tissue wrapping model in vitro Many investigators have designed 3D osteogenic bioreactors utilizing different sources of cells and types of scaffolds [23, 36, 37]. However, the osteogenic transformation of fibrous tissue in vitro is conceived impossible owing to the lack of a blood supply [20]. This study attempted for the first time to establish a tissue-scaffold complex in vitro that would support tissue survivability ex vivo and cast light on inducing de novo bone formation over a long culturing period, and is meant to replicate the normal in vivo experimental environmental conditions of most known extra skeletal bone inductive models [12, 13, 16, 35].

The abdominal skeletal muscle tissue of adult male Fischer 344/DuCrl rats was utilized, where macro-/microporous $\beta$-TCP/HA were either wrapped in the tissue harvested or where $\beta$-TCP/HA devices were first implanted in non-harvested muscle pouch within heterotopic sites, the standard experimental form to test new bone induction in vivo, and then excised before being cultured in vitro (Fig. 1). Implantation duration was limited to maximum of $30 \mathrm{~min}$ to prevent advanced tissue decay. In order to test the cell survival capacity of these two models, we extended the culturing time up to 30 days, where no evidence, to our knowledge, has yet reported on culturing muscle tissue ex vivo for more than 30 days. The tissue thickness was $\sim 1500 \mu \mathrm{m}$ in the wrapping model and $\sim 500 \mu \mathrm{m}$ in the pouch model. In the tissue wrapping model, no gene expression data could be generated for the 30 -day in vitro $\beta$-TCP/HA wrapped in skeletal muscle tissue from rats (Fig. 2i, Fig. $5 \mathrm{c}$ ), as the tissue became necrotic, gradually losing the original tissue structure with fading of nuclei preventing successful extraction of mRNA to be available for quantitative real-time polymerase chain reactions (qRT-PCR) analysis (Fig. 2a-c, Fig. 3a-d). On the other hand, single cells on $\beta$-TCP/HA devices pouched in the skeletal muscle survived the 30 -day in vitro culturing process (Fig. 2d-f, i, Fig. 3e-h), with no bacterial contamination in the culturing system (Fig. 4). Furthermore, histological analysis showed a consistent tissue survival around the scaffold in the pouch model up to 30 days, with ongoing tissue necrosis in the wrapping model over time.

With the goal of defining the difference of gene expression pattern between these two models and evaluate which method provides better tissue survival with possible osteogenic tendencies we then compared the qRT-PCR data between them. The tissue wrapping model, only at day 15 in vitro showed an up-regulation of tissue survival and angiogenesis markers including vascular epithelial growth factor $\alpha$ (VEGF-A) and collagen type 4 subunit 1 (COL4A1) and transforming growth factor $\beta 1$ (TGF- $\left.\beta_{1}\right)$ (Fig. 2h), whereas $\beta$-TCP/HA bioceramics pouched in abdominal skeletal muscle tissue of rats showed a considerable increase in angiogenesis and endothelial tissue formation genes expression at all time points (Fig. 2 g-i). For osteogenic differentiation markers, only bone morphogenetic protein 2 (BMP-2) up-regulation was noticed at day 5 in the wrapping model (Fig. 5a), while both runt-related transcription factor 2 (RUNX-2) and BMP-2 were steadily up-regulated over time in the pouch model and expressed at higher levels than in the wrapping model at day $30(P<0.01)$ (Fig. 5a-c). These results suggest better tissue survival in vitro in a tissue pouch model.

\section{Maintenance of vascular structure and stimulation of osteogenesis in tissue pouch models}

Upon demonstrating better tissue survivability and growth in the tissue pouch model through histology and gene expression patterns representative of cytoproliferation and differentiation supporting new tissue formation, 

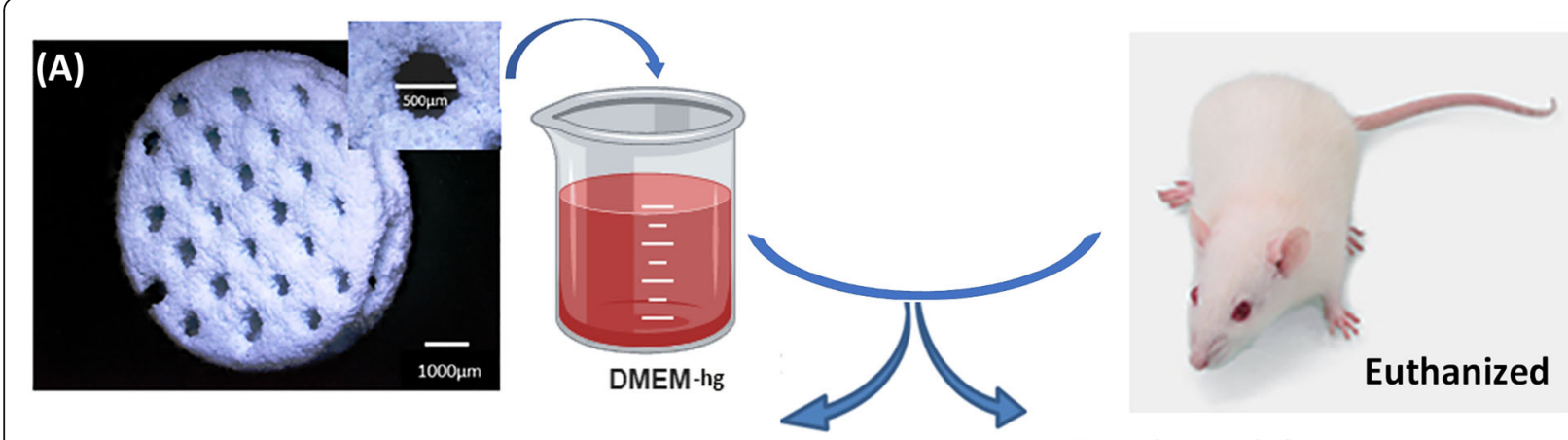

\section{Wrapping model}
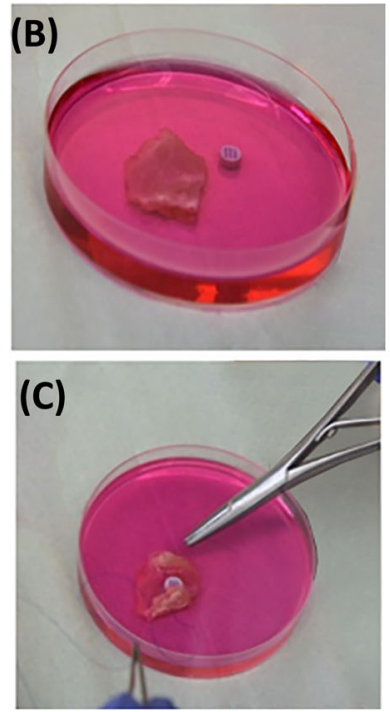

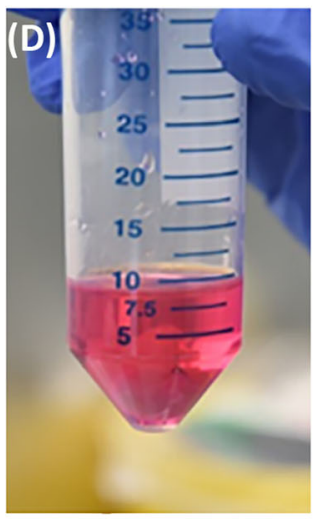

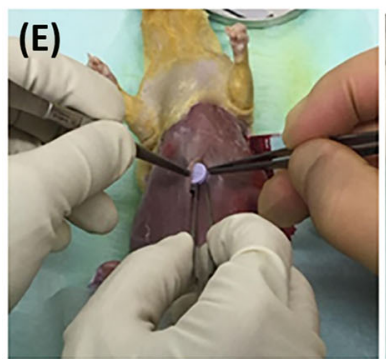

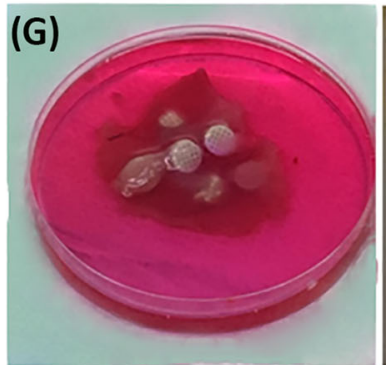

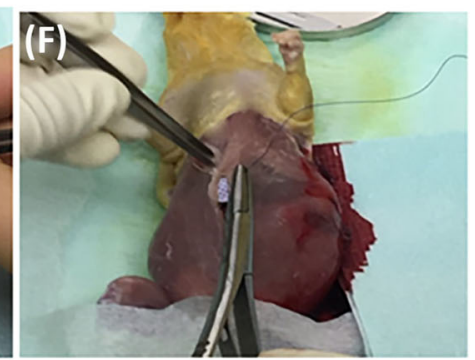

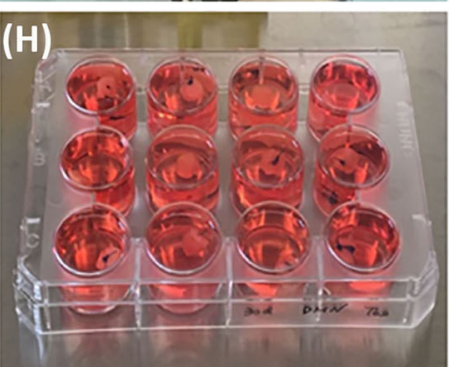

Fig. 1 In vitro wrapping and heterotopic implanted bioceramic pouch model methodology (a-h). The three-dimensional printed macro-porous $\beta$-tricalcium phosphate/hydroxyapatite ( $\beta$-TCP/HA) bioceramic devices (a), for the wrapping or pouch models, were placed in growth medium (DMEM), prior to either wrapping them in rat skeletal rectus abdominis muscle tissue (b-d), or implanting them first in heterotopic extra-skeletal rectus abdominis muscle sites (e-h) of euthanized rats. The implant sites with devices were then harvested, devices embedded in the muscle tissue excised, and subsequently placed in growth medium to be cultured for 5, 15 and 30 days in vitro. (All images within Fig. 1 originate from our own laboratory. Images were not taken from other sources)

the chronological change of $V E G F-A$ gene expression and protein production pattern up to 30 days of the culturing process and histological results at day 30 were assessed in the heterotopic pouch model (Fig. 6). This aimed to determine if a regulatory gene pattern could be identified and prove that this model indeed supports vascular structure maintenance and potential angiogenesis.

In $\beta$-TCP/HA bioceramic devices muscle pouch model, the best up-regulated genes were COL4A1, $V E G F-A$ and $T G F-\beta_{1}$ at day 15 and day 30, whilst at day 5 it was COL4A1, BMP-2 and VEGF-A (Fig. 2, Fig. 5). In short, COLAA1 and VEGF-A were highly up-regulated at all time points, whilst a marked high expression of $B M P-2$ occurred at day 30 compared with muscle tissue alone $(P<0.05)$ (Fig. 7i). Our findings, in the gene expression aspect, suggest that the bioceramic devices implanted in the muscle pouch support vessel survival and potential angiogenesis when cultured under normal in vitro growth conditions with limited osteogenic tendencies present, especially 30 days after treatment.

\section{Tissue pouch models initiate osteogenic morphogenesis ex vivo}

Histological sections of $\beta$-TCP/HA bioceramic devices wrapped in rat abdominal skeletal muscle tissue clearly showed a thin layer of fibrous-like tissue lining the interface between muscle tissue and scaffold at day 5 (Fig. 2a). In contrast, fibrils and cells were released from the injured muscle fibers and attached to the interface of the scaffold (Fig. 2d). Successively, a noticeable increase of the volume of necrotic muscle fibers was observed at day 15 in the bioceramic muscle tissue wrapped model (Fig. 2b), with limited numbers of condensed nuclei containing fibers sparsely distributed within ECM at the 


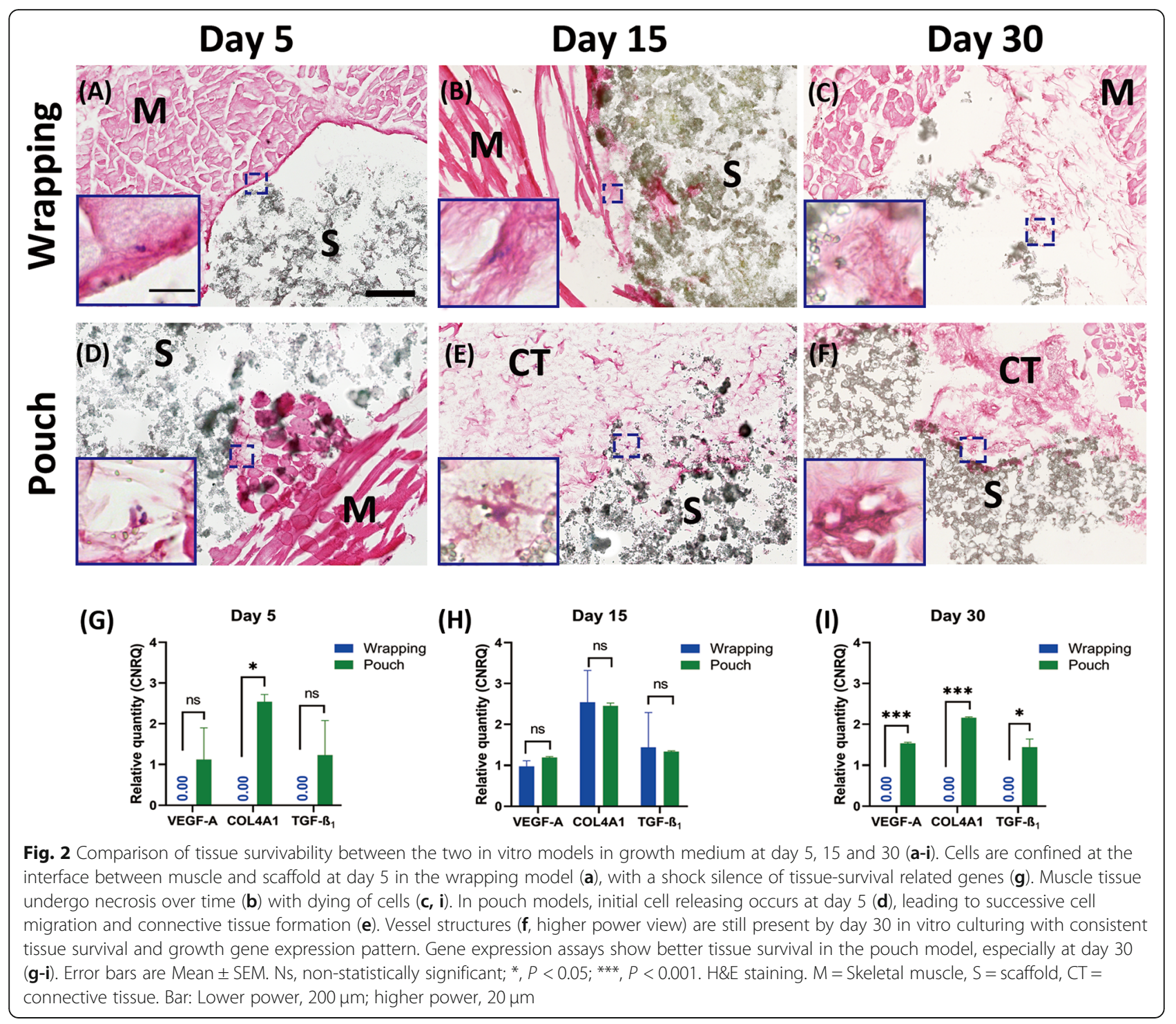

periphery of the devices. In contrast, muscle tissue of the bioceramic devices in the heterotopic pouch model, at day 15, appeared to actively "invade" and undergo a transformation, into connective tissue (Fig. 2e) that was clearly visible at the tissue to scaffold microporous interface and could partially be observed lining the macroporous hole-like structures of the scaffold (Fig. 2e higher magnification view). By day 30, in contrast to tissue pouched bioceramic devices, as represented in Fig. 2f, tissue survival was compromised in the wrapping model, where the muscle tissue on the periphery of the bioceramic device was observed to undergo a type of fragmentation, discontinuing fibrous tissue growth at the muscle to device interface (Fig. 2c), without any presence of living cells within the scaffold (Fig. 2c higher magnification view). Contrarily, for the 30 days heterotopic pouch model group, the muscle tissue was seen breaking down
(Fig. 7c and g), yet obviously supporting connective tissue that was observed invading, although mainly at the periphery, into the macroporous superstructure of the $\beta$ $\mathrm{TCP} / \mathrm{HA}$ devices (Fig. $7 \mathrm{~d}$ and $\mathrm{h}$ ), with fibrils also appearing to interact with the particles of the porotic bioceramic scaffold. No cells or tissues pertaining to bone formation could be visualized. Subsequently, during muscle tissue degeneration, cells within and between the muscle tissue fibers were released and appeared to be migrating into the scaffold together with the extracellular matrix (Fig. 7a, c and d). Certain transitional zones showed some signs of a collagen-osteoid-like matrix forming near the connective tissue to porous superstructure interphase of the device (Fig. 7e and h). These results indicated that cell migration could be initiated as early as day 5 , being supported up to 30 days by connective tissue in the tissue pouch model, with limited 

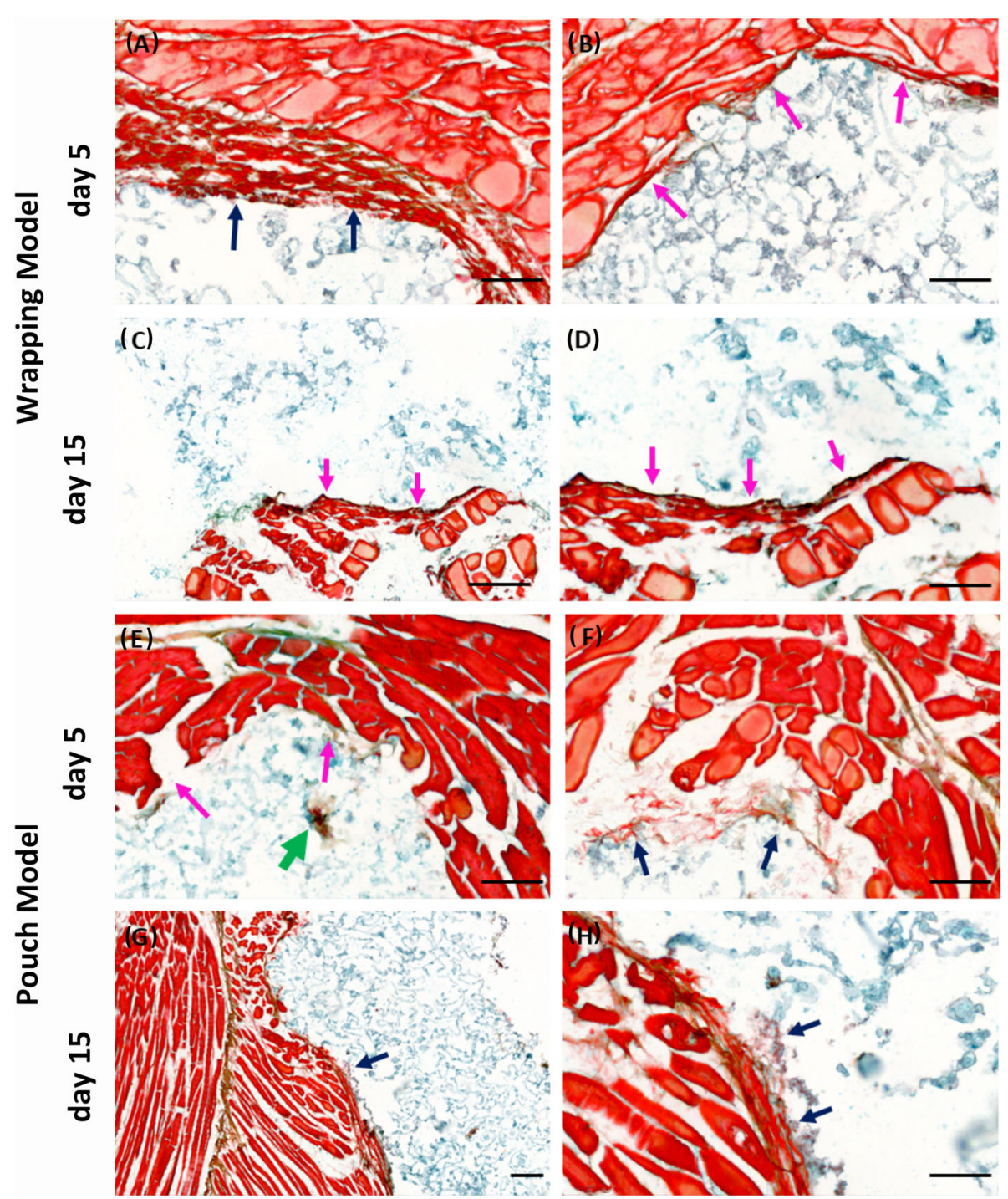

Fig. 3 Morphology and tissue response to devices in wrapping models and pouch models at day 5 and day 15 (a-h). A considerable amount of fibrils were seen forming into the device (a, $\mathbf{f}$; blue arrows) with some collagen-osteoid formation (green arrow) noticeable at days 5 , while the self-adaptation of tissue at the periphery of device was observed in both models (b, e; pink arrows). In contrast, to tissue implanted heterotopically ( $\mathbf{g}, \mathbf{h}$; blue arrows) the survivability of tissue was compromised in the tissue bag model at days 15 , where the muscle tissue on the periphery of the bioceramic device was observed to undergo a type of fragmentation, discontinuing fibrous tissue formation at the interface of the muscle and device ( $\mathbf{c}, \mathbf{d}$; pink arrows). Movat pentachrome staining was utilized to assess for collagen associated with chondrogenesis and osteogenesis, elastic fibers, muscle and connective tissue. Bars: A, B, D, E, F and $\mathrm{H}=100 \mu \mathrm{m} ; \mathrm{C}$ and $\mathrm{G}=200 \mu \mathrm{m}$

formation of collagen-osteoid-like matrices at the peripheries of the porous device.

\section{Discussion}

Developing a new technology that can fully replicate, synthetically, an in vivo environment in vitro, however challenging, is attractive as it would allow for more efficient testing on par with the physiological reality of the clinical setting. It is expected that such medically supportive platforms would deliver faster and superior results with reduced costs whilst allowing for more accurate prediction and therapeutic models to be developed for a clinical setting [38]. Whilst one solution to this problem has been the emergence of bioreactor platforms $[10,11,39,40]$ that have a limited capacity at replicating some in vivo processes, developing a synthetic system that can fully replicate the supra-organ of bone(s), let alone induce bone formation in vitro, with its plethora of varying proteins arranged geometrically within the 3D superstructure and assortment of cellular entities [40, 41] remains perhaps the most challenging prospect for tissue engineering regenerative sciences with only the neurological complexities of 


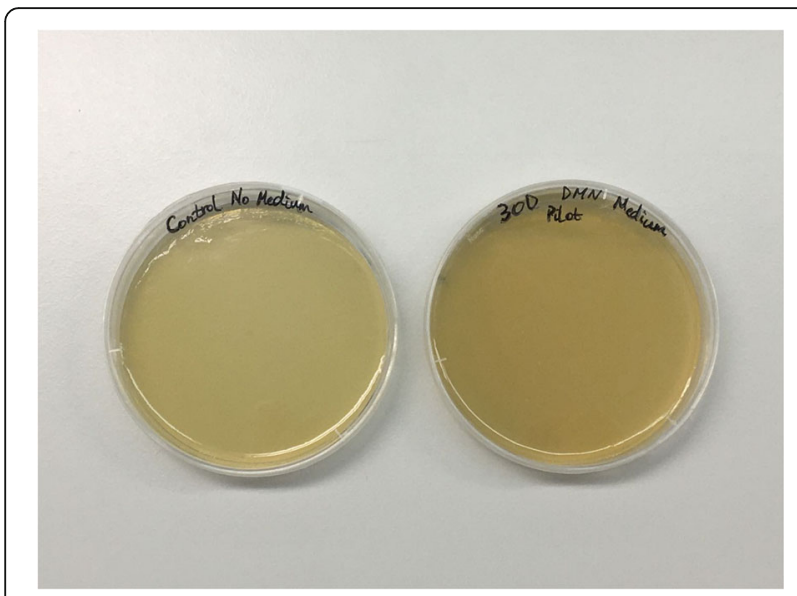

Fig. 4 Microbiological culture results of the 30-day culturing medium with a pouch model. No microbial contamination is detected in the 30-day culturing medium with a pouch model (right plate)

the brain surpassing this endeavor. In the hereby presented pilot study, the first nascent steps towards developing such a bone inductive/formative environmental reality in vitro was attempted. Systematic studies can be further developed and improved to produce in vitro bone formation of any skeletal bone in view of clinical applications.

The bone induction principle dictates that soluble molecular signal(s) combined with an insoluble substratum are critical for the initiation and formation of de novo bone tissue formation in vivo [13, 42]. The prerequisite, in order to facilitate proper bone formation, is an adequate vascular supply, formed either by vasculogenesis and/or angiogenesis, with vessel structures invading the macro- and microporous superstructure of a device and bringing vital stem cells, nutrients, amino acids, protein signals and other resources. This would culminate in new endothelial tissue invasion into the confines of the substratum, supplying nutrients necessary for subsequent new bone tissue formation [20, 43, 44]. However, what happens to an ex vivo tissue culture model when such prerequisites are not present. Can a tissue at all survive an extended culture period let alone support new tissue morphogenesis?

Previous research has shown that for complex tissues to properly survive in vitro they require certain conditions and special applications to survive, such as electrical or biomechanical stimulation [24]. Survival is limited, in which long term studies ultimately leads to tissue degeneration in which diffusion of nutrients and tissue building resources cannot reach the relevant cells as the vascular capillary system collapses [45]. This limits the tissue development in vitro to a critical size that permits nutrient diffusion, in particular the thicker a tissue ultimately becomes in vitro will generate survival issues. Indeed, in the present study, COL4A1 was originally chosen as it is a well-known biomarker for angiogenesis, where it is critical in the basement membrane formation of new capillaries and partially also in endothelial tissue development [14, 15, 17]. VEGF-A was included after interest was aroused at whether angiogenesis could also be developed, as it is known to support the endothelial tissue formation and act as a paracrine signaling molecule on the development and proliferation of endothelial cells [46]. Interestingly, qRT-PCR analysis and histological observations in our proof-of-concept study revealed that COLAA1 and VEGF-A was only briefly up-regulated within the wrapping model at day 15 , after which the tissue died off in tissue culture. On the other hand, the $\beta$-TCP/HA device pouched in abdominal skeletal muscle sites, harvested and then cultured in vitro, showed a consistent and almost regulatory pattern of endothelium proliferation and/or angiogenesis up to 30 days at either transcriptional or translational level. This could also, at least for connective and endothelial-like tissue formation and invasion into the $\beta$-TCP/HA bioceramics, were validated histologically (Fig. 6). Here new connective tissue formation was histologically apparent by day 30 , invading the
(A)

Osteogenic differentiation related genes expression data on day 5

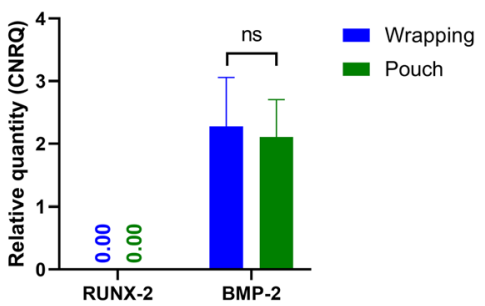

(B)

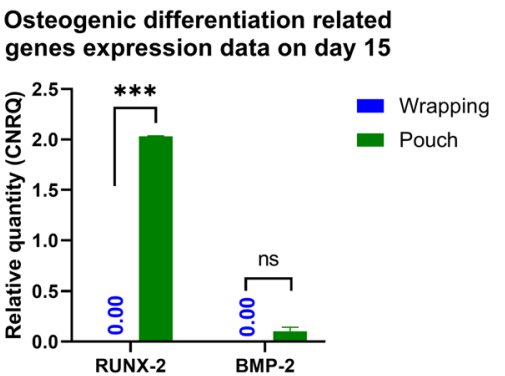

(C)

Osteogenic differentiation related genes expression data on day 30

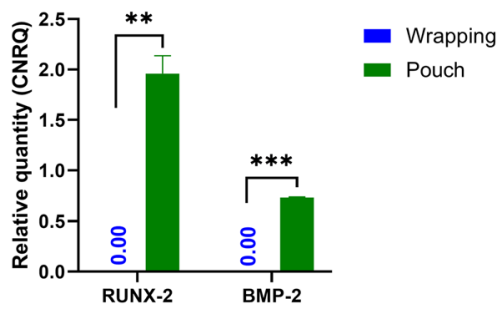

Fig. 5 Chronological osteogenic-related gene expression pattern in both wrapping and pouch model (a-c). Pouch models showed superior osteogenic differentiation capacity at day 15 (b) and 30 (c) comparing wrapping models. Error bars are Mean \pm SEM. Ns, non-statistically significant; ${ }^{* *}, P<0.01 ;{ }^{* *}, P<0.001$ 

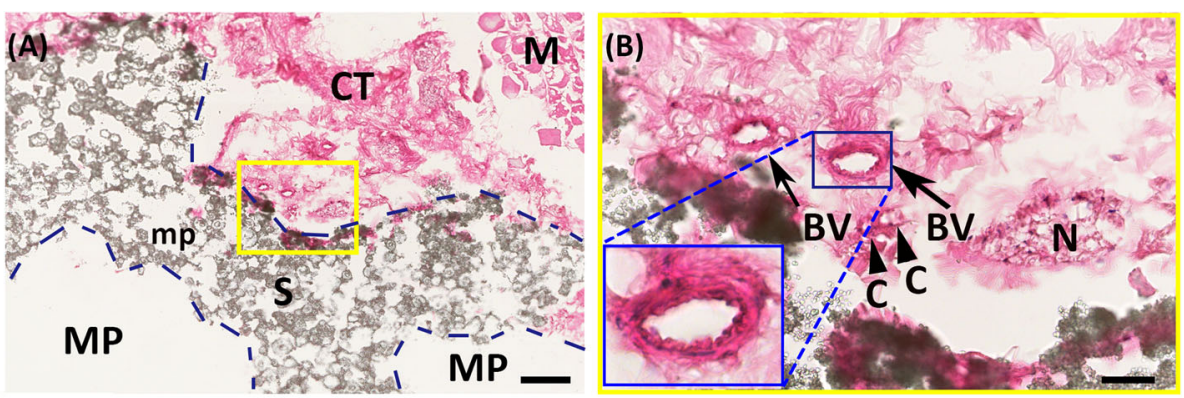

(C)

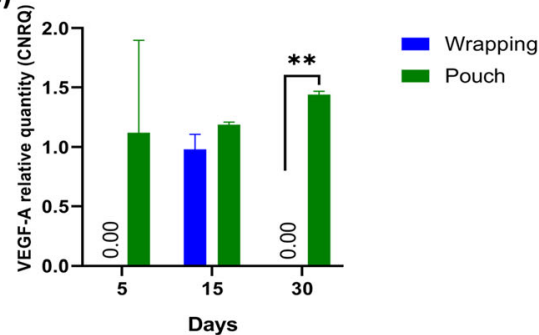

(D)

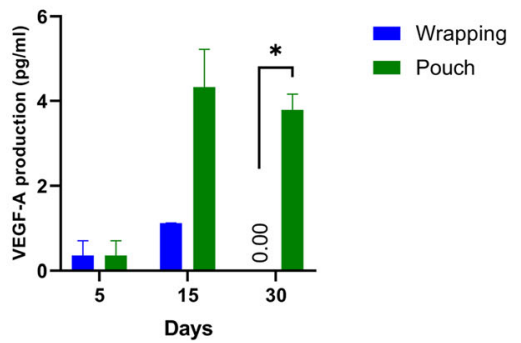

Fig. 6 Maintenance of vascular structure and potential of angiogenesis in tissue pouch models up to 30 days (a-d). Connective tissue grows into the macropore of the scaffold at the periphery (a, dotted lines show the contour of the macropores), with neurovascular bundle still surviving by 30 days (b). Both transcriptional (c) and translational (d) results suggest the maintenance of angiogenesis capacity with a pouch model by 30 days, whereas the capacity is lost with a wrapping model $\left(P<0.01\right.$ and 0.05 , respectively). Error bars are Mean \pm SEM. ${ }^{*}, P<0.05 ;{ }^{*}, P<0.01$. H\&E staining. $\mathrm{M}=$ Skeletal muscle, $\mathrm{S}=$ scaffold, $\mathrm{CT}=$ connective tissue, $\mathrm{MP}=$ macropores, $\mathrm{mp}=$ micropores, $\mathrm{BV}=\mathrm{blood}$ vessel, $\mathrm{N}=$ nerve, $\mathrm{C}=\mathrm{capillary}$. Bar: A, $200 \mu \mathrm{m} ; \mathrm{B}, 50 \mu \mathrm{m}$

macroporous superstructure of the devices, near the peripheries only. This deviation between the wrapping and pouch models clearly reflects the criteria of diffusion of nutrients across certain tissue thickness in vitro. On one hand, the wrapping model with a muscle thickness of almost $1500 \mu \mathrm{m}$ prevented proper nutrient flow to the intrinsic muscle cells near the biomaterial-cell interphase. This on the other hand was not the case for the pouch model in which the muscle tissue thickness from medium-cell and cell-biomaterial interphase was only $500 \mu \mathrm{m}$ thick. Moreover, whilst true osteogenesis eluded our investigations, as this was not a central aim as yet at this point, the gene expression level of $R U N X-2$ increased considerably at both day 15 and 30 in the pouch groups with also positive up-regulation of $B M P-2$ and $T G F-\beta_{1}$. This suggests that the presently utilized organoid pouch model has the potential to induce new bone formation at an in vitro cell culturing level, as it was demonstrated to do in vivo in various animal models $[16,23,27,47,48]$. Again, possibly because of the stem cell availability and absent tissue morphogenesis due to a lack of an active blood supply that would normally bring in extra progenitor stem cells and even monocyte/ macrophages critical for osteoclastogenesis [16] bone formation was retarded. Multiple studies reported that interconnection pathways have a strong impact on new tissue development, with incomplete and undersized pore interconnection limiting efficient connective tissue infiltration and blood vessels invasion into the scaffold [34]. However, in the present study, the average diameter of the interconnection pathway was $\sim 40 \mu \mathrm{m}$, indicating the limited capacity for sound tissue and vascular invasion. This could explain why connective tissue formation and vascular survival were only observed in this study at the peripheral macropores with diameter larger than $500 \mu \mathrm{m}$. This leaves new strategic avenues open to improve the responsive signals in the system. Follow-up experiments need to be considered to investigate this aspect further and see how other substrata would affect tissue morphogenesis in vitro.

However, aside from the initial validations of the in vitro organoid pouch model as a tissue model to be utilized for further investigations with good survival chances as well as partial osteogenic support combined with angiogenic responses, our study serendipitously revealed new connective tissue formation and endothelial tissue survival at the peripheral region of the heterotopic pouch implanted $\beta$-TCP/HA devices. This suggests that in vitro blood vessel had survived the long-term culture period with resident cells producing the necessary signals that are required for tissue survival with the potential to angiogenesis that could support connective tissue ingrowth into the scaffold and the subsequent osteogenic differentiation of mesenchymal stem cells located within the connective tissue (Figs. 6 and 7). We postulate that the surrounding tissue in heterotopic sites are 

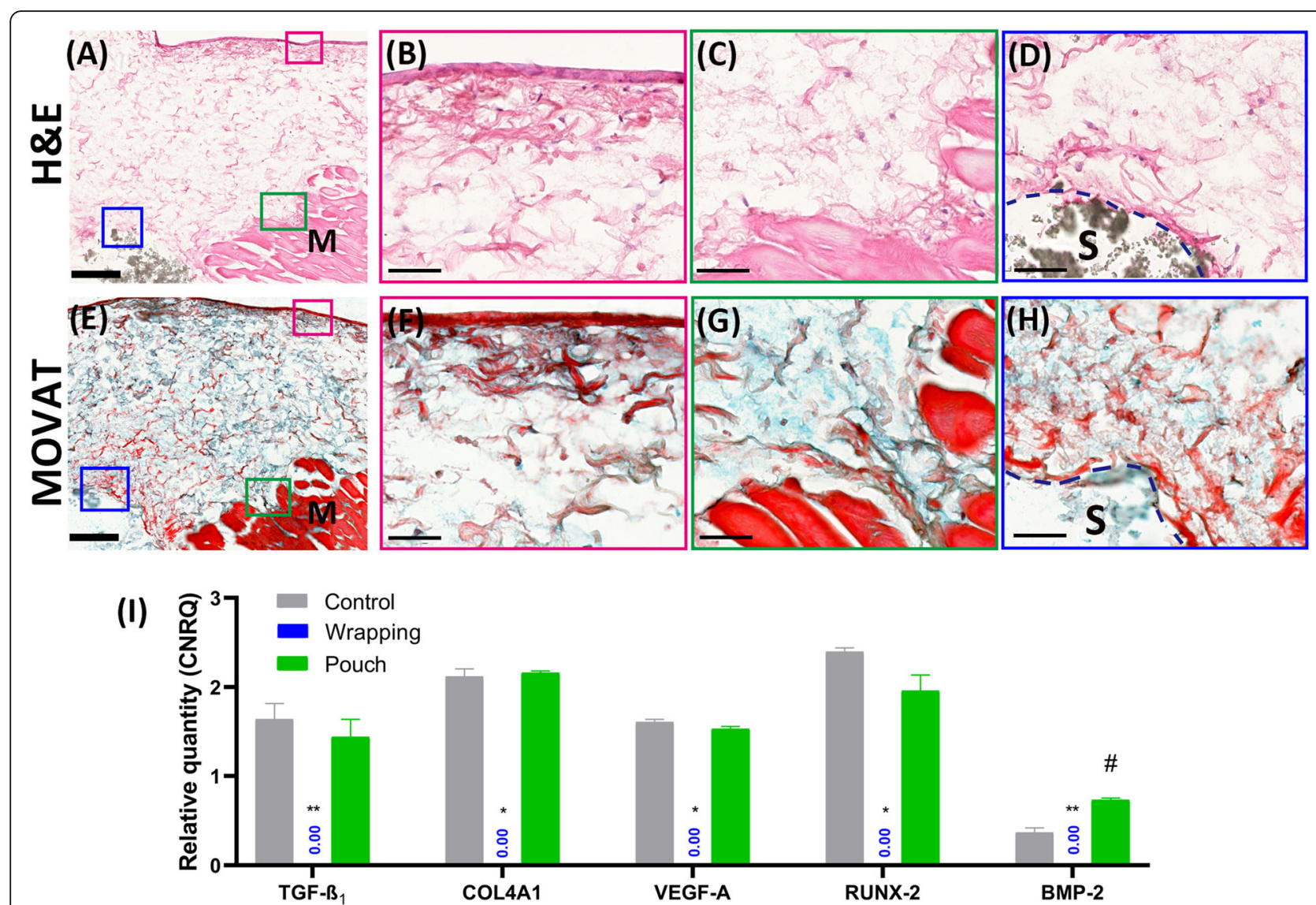

Fig. 7 Representative morphology and tissue response to devices in pouch models at day 30 (a-i). Extensive connective tissue forms (a and $\mathbf{b}$ ) around the scaffold, with comprehensive mucin deposition (e in blue) and fibrils (e in red) evenly distributed in between, consistent with the gene expression pattern showing proliferation and angiogenesis (i). A tissue layer forms at the interface contacting medium (b and $\mathbf{f}$ ), where fibrous-like cells line at the surface of tissue $(\mathbf{b})$, producing condensed fibers ( $\mathbf{f}$ in red) underneath. Cells releasing from muscle fiber (c) migrate within the mucin-fibril rich extracellular matrix $(\mathbf{g})$ towards either outer layer or scaffold (d and $\mathbf{h}$ ). The osteoid (h, area in scarlet) mesh at the interface (dashed lines) between tissue and scaffold indicates the osteogenic transformation of the connective tissue, which is supported by BMP2 gene expression results $(P<0.05)$. Error bars are Mean \pm SEM. ${ }^{*}, *, P<0.05 ;{ }^{* *}, P<0.01$. H\&E staining $(\mathbf{a}-\mathbf{d})$; Movat pentachrome staining (e-h). $\mathrm{M}=$ Skeletal muscle, $\mathrm{S}=$ scaffold. Bar: A and E, $200 \mu \mathrm{m} ; \mathrm{B}-\mathrm{D}, \mathrm{F}-\mathrm{H} 50 \mu \mathrm{m}$

actively engaged in the formation of specific connective and/or endothelial tissue formation rather than simply providing a signal that facilitates an immunological response or acting as a stem cell reservoir to sustain the metabolic formation of new bone by induction with an insoluble substratum [13, 16-18, 47].

Various investigations into in vitro metabolistic effects of cells removed from their natural environment and cultured within an ex vivo system clearly re-iterate that cells lose their homeostatic state where critical essential amino acid building blocks, normally available for protein synthesis, suddenly disappear. This greatly limits efficient protein translation [26], including losing critical energy production requirements to fuel necessary anabolic activities to support formation of complex ECM components [49]. Catabolic reactions using glucose, adipose tissue or proteins are a necessary requirement for the survival of any cell, let alone a tissue. In vitro systems cannot adequately replicate these reactions and might prevent cellular in vitro tissue experiments from progressing past the generally accepted 30 -day culturing period limit $[45,50,51]$. After this, because of extensive proliferation of cells or tissues, the catabolic breakdown into basic components and energy might be insufficient to meet the anabolic synthetic requirements to maintain cells and/or tissues active in vitro and might therefore limit their capacity to form larger complex organs. However, in light of the histological results of the present study of the skeletal muscle pouched $\beta-\mathrm{TCP} / \mathrm{HA}$ bioceramic devices cultured in vitro at days 15 and 30, we hypothesize that the muscle tissue rescues the catabolic and anabolic homeostasis by behaving as a catabolic reservoir that breaks down into base components. Previous research by Brand (1997), Albert (2005) including Nelson and Cox (2005), have shown that as tissue degenerates in vitro it has the potential to release glucose, 
proteins but also critically essential amino acid that could be utilized as energy and building blocks by resident cells to support new tissue development, as seen at the peripheries of the bioceramic to muscle tissue interphase (Fig. 7) of the present study. This would allow resident stem cells to undergo differentiation and proliferation into the macroporous spaces of the bioceramic device, depositing new endothelial tissue matrix that could support vascular structures. There the culturing medium might act as a nutrient source to more effectively transport biochemical buildings blocks and nutrients into the confines of the device, providing the means for tissue survival. This form of cell differentiation and tissue repurposing or "hypertrophic tissue transformation" needs to be further validated and elucidated if it indeed is some type of tissue "recycling" modus or is simply an artefact of deterioration. Similarly, the benefit of a tissue organoid in vitro culturing system over standard cell culture modes still needs to be assessed further. We believe that the tissue organoid model might more efficiently support critical catabolic and anabolic mechanisms and assist in more complex cytological reactions, to help form more complex organ structures.

\section{Conclusions}

The present proof-of-concept study clearly showed that an organoid pouch model exhibits superior survivability and more consistent tissue growth in vitro compared with a wrapping model, thereby rendering the approach promising for follow-up bone inductive endeavors provided the correct material and/or signals are present to facilitate this reaction. However, whilst the in vitro tissue inductive model can support the development in part of an angiogenic response, the culturing system needs to be further supplemented and enhanced with either the relevant stem cells including monocytes/macrophages/osteoclasts uniting a synthetic circulatory system that would enable future in vitro models to function as an in vivo system would. Subsequently, differences in molecular signals between in vitro and in vivo pouch models, including macro and micro signals involved in new autogenous bone formation, still need to be determined that would enable such future models to fully replicate the in vivo environment ex vivo.

\section{Methods}

\section{Aims and study design}

The present pilot study explored the tissue behavior and cell survival capability within a new in vitro skeletal muscle tissue-based biomaterial organoid bioreactor.

Eighteen 3D printed $\beta$-tricalcium phosphate/hydroxyapatite devices were either wrapped in a sheet of rat rectus abdominis muscle tissue $(n=9)$ or first implanted in a heterotopic rectus abdominis muscle pouch $(\mathrm{n}=9)$ that was then excised and cultured in vitro for up to 30 days. Normal rectus abdominis muscle tissue without implants, uncultured, served as the endogenous control to which all samples were compared to. Specimens were harvested at 5 days, 15 days and 30 days $(n=3$ per time point), respectively and underwent qRT-PCR and histological analyses. Supernatants of tissue cultures were assayed for angiogenic/vasculogenic protein production, fresh medium was the control.

\section{D printed $\beta$-tricalcium phosphate/hydroxyapatite devices $(\beta-T C P / H A)$ devices}

Eighteen devices were provided by BioMed Center Innovation gGmbH (Bayreuth, Germany). According to the BioMed Center, the 3D-printed $\beta-\mathrm{TCP} / \mathrm{HA}$ bioceramic devices (Fig. 1a) had been manufactured using a mixture of tri-calcium phosphate and hydroxyapatite powders (Merck, Kenilworth, NJ, USA)) at a ratio of 40\%:60\%, respectively. The mixture had previously been spray-nozzle granulated from a water-based slurry with addition of organic dispersing and binding agents using a custom spray-dryer (Trema, Kemnath) and cut off at $100 \mu \mathrm{m}$ using a classing sieve (Retsch, Haan, Germany). The lower fraction of the granulate was coated with organic adhesion-improving agents by means of fluidized bed coating; the final printing powder had size distribution values of $\mathrm{d} 10=34.87 \mu \mathrm{m}, \mathrm{d} 50=61.86 \mu \mathrm{m}$ and $\mathrm{d} 90=$ $93.33 \mu \mathrm{m}$. After mixing the powder with a combination of organic additives (trade secret), the scaffolds were then printed out in a Z310 3D-Printer (3D Systems, Rock Hill, USA) using the standard colorless ink provided with the printer. After de-powdering, the scaffolds were sintered at $1250^{\circ} \mathrm{C}$, producing a solid, organic-free, porous bioceramic device with macroscopic pore channels $(670.52+/-97.60 \mu \mathrm{m})$ resulting from printing design and smaller internal pores $(80.95+/-23.38 \mu \mathrm{m})$ as described above. The devices were then allowed to cool, after which they were cleaned using deionized water, packed and sterilized by vacuum pulse autoclaving.

\section{Skeletal muscle-based biomaterial culturing models}

Commercially available, four adult male Rattus norvegicus Fischer 344/DuCrl rats (Charles River Laboratories, Sulzbach, Germany), were utilized in the pilot study, and equally split between the two tissue models. Animals were euthanized with an overdose of isoflurane (Abbot, Chicago, USA). This was done in accordance to the rules and regulations of the Animal Protection Laboratory Animal Regulations (2013), European Directive 2010/63/ EU and approved by the Animal ethics research committee (AESC) of the Ludwig Maximillian's University of Munich (LMU), Bavaria, Germany Tierschutzgesetz $\$ 1$ / $\$ 4 / \mathbb{\$} 17 \quad$ (https://www.gesetze-im-internet.de/tierschg/ TierSchG.pdf) with respect to animal usage for pure 
tissue or organ harvest only. No muscle tissue was purchased commercially or otherwise. All muscle tissue utilized in the study was extracted by our laboratories from commercially bought adult male Rattus norvegicus Fischer 344/DuCrl rats (Charles River Laboratories).

Two skeletal muscle tissue biomaterial-based models were designed and tested:

\section{Tissue wrapping model}

For the tissue wrapping model, $n=9 \beta$-TCP/HA devices, were first immersed in normal growth medium composed of Dulbecco's modified Eagle medium-high glucose (DMEM-hg) (Biochrom GmbH, Berlin, Germany), $40 \mathrm{IU} / \mathrm{mL}$ penicillin (Biochrom $\mathrm{GmbH}$ ) and $40 \mathrm{IU} / \mathrm{mL}$ streptomycin (Biochrom $\mathrm{GmbH}$ ).

Two F-344 adult male rats (Charles River Laboratories) were euthanized under sterile conditions, the rectus abdominis skeletal muscle tissue harvested, placed in normal DMEM-hg after which 3D printed $\beta$-TCP/HA devices were wrapped in the sheets of rectus abdominis muscle tissue (Fig. 1b-f). Nine $\beta$-TCP/HA devices were then wrapped with a skeletal rectus abdominis muscle sheet, and divided into 3 culturing periods set at 5, 15 and 30 days. Each culturing period contained 3 tissue bags. Rectus abdominis muscle tissue without $\beta$-TCP/HA devices was cultured in parallel to tissue bags and acted as controls. Medium was changed every 2 days. Fresh rectus abdominis muscle tissue was used in the normalization of qRT-PCR.

\section{Tissue pouch model}

Nine $\beta$-TCP/HA devices were prepared by placing them in normal growth medium as explained in the section of the tissue wrapping model. Rats were then euthanized under sterile conditions, $\beta$-TCP/HA devices were immediately implanted in intramuscular rectus abdominis muscle pouches created by sharp and blunt dissection (Fig. 1g-j). Once all $\beta$-TCP/HA devices had been implanted, rectus abdominis muscle tissue pouches with biomaterials were excised using $8 \mathrm{~mm}$ biopsy punches (PFM medical, Cologne, Germany). Nine rectus abdominis muscle pouches with $\beta$-TCP/HA were created, and divided into 3 culturing periods set at 5, 15 and 30 days. Each culturing period contained 3 tissue pouches. Rectus abdominis muscle tissue without $\beta-\mathrm{TCP} / \mathrm{HA}$ devices were cultured in parallel to tissue pouches and acted as controls. Medium was changed every 2 days. Fresh rectus abdominis muscle tissue was used in the normalization of qRT-PCR.

After the allotted culturing period, specimens with $\beta$ TCP/HA devices were harvested and cut midways, with one-half flash frozen in liquid nitrogen for qRT-PCR assays and the other half fixed in 4\% paraformaldehyde (Microcos GmbH, Garching, Germany) to be processed for histological and histomorphometric analysis.

\section{Bacterial contamination assay}

The 30-day organoid pouch model devices, during histological analysis, were observed containing a filamentous fibrous-like material. To exclude the likelihood that the fibrous like material was not of bacterial origin and in fact fibrin fibrils, the medium collect for quantitative protein analysis from these samples was subjected to a bacterial contamination test. Under sterile conditions collected culture medium was plated out on a standard Luria Broth Agar (LA) plates (1 g Tryptone, $1.5 \mathrm{~g}$ Technical agar, $0.5 \mathrm{~g}$ Yeast extract, $0.5 \mathrm{~g} \mathrm{NaCl}$ (all (Sigma-Aldrich)) in $100 \mathrm{ml}$ $\mathrm{dH} 2 \mathrm{O}$ ), with a normal LA plate with fresh DMEM-hg (Biochrom $\mathrm{GmbH}$ ) medium set as control. After $72 \mathrm{~h}$ of incubation at $37^{\circ} \mathrm{C}$ with $5 \% \mathrm{CO}$, plates were assessed for bacterial colony formation by one blinded analyst (Yan Chevalier).

\section{QRT-PCR}

QRT-PCR was performed to determine the relative gene expression quantity of tissue growth related genes especially angiogenesis and endothelial tissue formation genes, VEGF- $A$, COL4A1 and TGF- $\beta_{1}$ including known osteogenesis signaling and structural markers, specifically RUNX-2 and BMP-2.

Specimen fragments for qRT-PCR were ground to powder in the presence of liquid Nitrogen. Total RNA was then isolated using a modified RNA Trizol extraction procedure (Chomczynski \& Mackey, 1995). Briefly, $1 \mathrm{ml}$ Trizol (Invitrogen, San Diego, CA, USA) was added to the powderised tissue, where through the addition of chloroform (Sigma-Aldrich) the aqueous RNA containing phase was transferred to Isopropanol (Sigma-Aldrich). RNA was then pelleted out in an overnight centrifugation step at $4{ }^{\circ} \mathrm{C}$, which were then washed with $75 \%$ ethanol dried and resuspended in $32 \mu \mathrm{l}$ RNase free water. The concentration of the RNA was determined using a NanoDropTMLite (Thermo Scientific, Waltham, USA) and quality assessed with a Bioanalyzer 2100 (Agilent Technologies, CA, USA). RNA integrity numbers lower than 8 were not accepted. RNA was then reverse transcribed into complementary DNA (cDNA) using the QuantiTect Reverse Transcription cDNA Synthesis Kit (Qiagen, Hilden, Germany).

QRT-PCR was then performed, in duplicate with FastStart Essential DNA Green Master (Roche, Basel, Switzerland) in a final reaction volume of $10 \mu$ l, using a LightCycler 96 thermocycler (Roche). Each reaction contained 10 ng cDNA; 2x FastStart Essential DNA Green Master and $10 \mu \mathrm{M}$ of each primer (Table 1). Primers were designed using Integrated DNA Technologies PrimerQuest Tool (https://eu.idtdna. com/Primerquest/Home/Index). Use of GeNorm (http:// medgen.ugent.be/ jvdesomp/genorm/) established that ribosomal protein large PO (RPLPO), succinate dehydrogenase complex subunit A (SDHA), RNA polymerase II subunit E 
Table 1 Gene primer sequences for test and reference genes

\begin{tabular}{|c|c|c|}
\hline Gene & Forward Primer (5'-3') & Reverse Primer (5'-3') \\
\hline VEGF-A & CTACCAGCGCAGCTATTG & GATCCGCATGATCTGCATAG \\
\hline COL4A1 & CTGGGAATCCCGGACTT & GGGATCTCCCTTCATTCCT \\
\hline$T G F-\beta_{1}$ & TTAGGAAGGACCTGGGTT & ACCCACGTAGTAGACGATG \\
\hline$B M P-2$ & GGAAGTGGCCCACTTAGA & TCACTAGCAGTGGTCTTACC \\
\hline$R \cup N X-2$ & CCCAAGTGGCCACTTAC & CTGAGGCGGTCAGAGA \\
\hline RPLPO (reference) & CAACCCAGCTCTGGAGA & CAGCTGGCACCTTATTGG \\
\hline SDHA (reference) & GCGGTATGACACCAGTTATT & CCTGGCAAGGTAAACCAG \\
\hline POLR2E (reference) & GACCATCAAGGTGTACTGC & CAGCTCCTGCTGTAGAAAC \\
\hline TBP (reference) & TAACCCAGAAAGTCGAAGAC & CCGTAAGGCATCATTGGA \\
\hline
\end{tabular}

(POLR2E) and TATA binding protein (TBP) were the most appropriate internal reference genes to use in this experiment. All amplified PCR products underwent Sanger sequencing (GATC Biotech, Cologne, Germany) and were then analyzed utilizing nucleotide analysis (https://blast.ncbi. nlm.nih.gov/Blast.cgi? PAGE_TYPE = BlastSearch) to confirm that the correct sequence had been amplified. QRTPCR thermocycling parameters included a pre-incubation of $3 \mathrm{~min}$ at $95^{\circ} \mathrm{C}$, followed by a three-step amplification program of 40 cycles consisting of a denaturation, annealing and extension step set at $95^{\circ} \mathrm{C}$ for $10 \mathrm{~s}, 60^{\circ} \mathrm{C}$ for $15 \mathrm{~s}$ and $72{ }^{\circ} \mathrm{C}$ for 30s, respectively. Relative gene expression was normalized against four reference genes. Gene expression from the harvested tissue/device models was normalized to the four reference genes and fresh abdominal skeletal muscle tissue using the Qbase+ software (http://www.biogazelle.com). Gene expression results were represented as mean calibrated normalized relative quantities (CNRQs) \pm standard error, which reflect the $\log _{10} 2^{-\Delta \Delta C t}$.

\section{Histological evaluation}

Specimens were fixed in $4 \%$ paraformaldehyde (Microcos $\mathrm{GmbH}$ ) for $24 \mathrm{~h}$ after which they were processed for paraffin wax embedding. Prior to cutting $10 \mu \mathrm{m}$ sections the surface of each paraffin block was decalcified [52]. In order to validate our gene expression patterns with respect to tissue survivability within the two tissue models, histological sections were stained using either the hematoxylin (Morphisto GmbH, Frankfurt, Germany) and eosin (H\&E) staining [53] (Morphisto $\mathrm{GmbH}$ ) or the Movat pentachrome staining [54] (Morphisto $\mathrm{GmbH}$ ). Stained sections were subsequently analyzed under PreciPoint M8 microscope (PreciPoint, Freising, Germany).

\section{Quantitative angio-/vasculogenic protein assays}

The amount of VEGF-A produced by the two bioreactors and controls were determined using Magnetic Luminex ${ }^{\odot}$ Assays (R\&D systems, Minneapolis, USA). Supernatants of tissue cultures were harvested at 5 days,
15 days and 30 days for either the wrapping model specimens or the pouch model specimens and controls. Absolute VEGF-A content in supernatants were measured according to the manufacturer's instructions. Results were generated using $\mathrm{xPONENT}^{\oplus} 4.2$ for MAGPIX ${ }^{\oplus}$ Software (R\&D systems, Minneapolis, USA).

\section{Statistical analysis}

Data were analyzed using GraphPad Prism v8.0.1 (GraphPad Software, San Diego, USA). The results were represented as mean \pm standard error (SEM). Measurements were performed in triplicate or duplicate when no valid data could be obtained from one sample per group. The Mann-Whitney test was used to detect statistical differences with $\alpha=0.05$. Statistical significance was indicated by ns for no significance, * for $p<0.05$, ** for $p<0.01$ and $* * *$ for $p<0.001$.

\section{Abbreviations}

3D: Three dimensional; BMP-2: Bone morphogenetic protein 2; CNRQ: Calibrated normalized relative quantities; COL4A1: Collagen type 4 subunit 1; ECM: Extracellular matrix; H \& E: Hematoxylin and eosin; POLR2E: RNA polymerase I/ subunit E; QRT-PCR: Quantitative real-time polymerase chain reactions; RPLPO: Ribosomal protein large P0; RUNX-2: Runtrelated transcription factor 2; SDHA: Succinate dehydrogenase complex subunit A; TBP: TATA binding protein; TGF- $\beta_{1}$ : Transforming growth factor $\beta_{1}$; VEGF-A: Vascular epithelial growth factor a

\section{Acknowledgments}

The biomaterial was generously provided to us by Dr. Daniel Seitz and Mr. Florian Gaudig from the BioMed Center Innovation gGmbH in Bayreuth. We would like to thank Prof. Dr. Stefan Milz (Dept. of Anatomy, LMU Munich) for discussing the results with the authors. We would also like to thank our technical staff especially Mrs. Barbara Berens, Ms. Sandra Haßelt and Ms. Bärbel Schmitt for their technical assistance during the project.

\section{Authors' contributions}

$\mathrm{TH}$ performed the experiments, analyzed and interpreted parts of the gene expression data and constructed the layout and initial draft of the manuscript. JH revised the manuscript and supervised TH progress. YC revised the manuscript and was instrumental in providing key ideas, through biomechanical principles, that lead to the data interpretation. RK conceived and designed this work, co-supervised $\mathrm{TH}$, revised the manuscript content, interpreted the gene expression data and constructed the discussion of the manuscript including initial scientific English editing. All authors read and approved the final manuscript. RK takes responsibility for the integrity of the data analysis. 


\section{Funding}

This research was funded internally and from the China Scholarship Council, grant number 201606230235. The funding bodies were not involved in the design of the study, the collection of data, data analysis, in the interpretation of data and in writing the manuscript.

\section{Availability of data and materials}

The necessary algorithmic codes of the program GeNorm are readily available at (http://medgen.ugent.be/wjvdesomp/genorm/). All data, raw and processed, is readily available from the corresponding author on request.

\section{Ethics approval and consent to participate}

All experiments were done in accordance to the rules and regulations of the Animal Protection Laboratory Animal Regulations (2013), European Directive 2010/63/EU and approved by the Animal ethics research committee (AESC) of the Ludwig Maximillian's University of Munich (LMU), Bavaria, Germany Tierschutzgesetz $\$ 1 / \S 4 / \$ 17$ (https://www.gesetze-im-internet.de/tierschg/ TierSchG.pdf) with respect to animal usage for pure tissue or organ harvest only. No muscle tissue was purchased commercially or otherwise. All muscle tissue utilized in the study was extracted by our laboratories from commercially bought adult male Rattus norvegicus Fischer 344/DuCrl rats (Charles River Laboratories, Sulzbach, Germany).

\section{Consent for publication}

Not Applicable.

\section{Competing interests}

The authors declare that they have no competing interest.

\section{Author details}

'Department of Orthopedics, Physical Medicine and Rehabilitation, University Hospital of Munich (LMU), Munich, Germany. ${ }^{2}$ Department of Orthopedics, Renji Hospital, School of Medicine, Shanghai Jiao Tong University, Shanghai, China

Received: 12 September 2019 Accepted: 21 January 2020

Published online: 31 January 2020

\section{References}

1. Amini AR, Laurencin CT, Nukavarapu SP. Differential analysis of peripheral blood- and bone marrow-derived endothelial progenitor cells for enhanced vascularization in bone tissue engineering. J Orthop Res. 2012;30(9):1507-15.

2. Anderer $U$, Libera J. In vitro engineering of human autogenous cartilage. J Bone Miner Res. 2002:17(8):1420-9.

3. Denayer T, Stöhr T, Van Roy M. Animal models in translational medicine: validation and prediction. New Horiz Transl Med. 2014;2(1):5-11.

4. Collier R. Rapidly rising clinical trial costs worry researchers. Can Med Assoc; 2009.

5. Havers C, Geuder MF. Osteologia nova: sive, Novae quaedam observationes de ossibus, et partibus ad illa pertinentibus: Apud Georgium Wilhelmum Kühnium; 1692.

6. Ollier L. Traité expérimental et clinique de la régénération des os et de la production artificielle du tissu osseux: V: Masson; 1867.

7. Senn N. On the Healing of Aseptic Bone Cavities by Implantation of Antiseptic Decalcified Bone. Am J Med Sci. 1889:98:219ß43.

8. Atef M, Osman AH, Hakam M. Autogenous interpositional block graft vs onlay graft for horizontal ridge augmentation in the mandible. Clin Implant Dent Relat Res. 2019;21:678.

9. Galindo-Moreno P, Avila G, Fernandez-Barbero JE, Mesa F, O'Valle-Ravassa F, Wang HL. Clinical and histologic comparison of two different composite grafts for sinus augmentation: a pilot clinical trial. Clin Oral Implants Res. 2008;19(8):755-9.

10. Martin I, Wendt D, Heberer M. The role of bioreactors in tissue engineering. Trends Biotechnol. 2004;22(2):80-6

11. Plunkett N, O'Brien FJ. Bioreactors in tissue engineering. Technol Health Care. 2011;19(1):55-69.

12. Urist MR, Strates BS. Bone morphogenetic protein. J Dent Res. 1971;50(6): 1392-406.

13. Sampath TK, Reddi AH. Dissociative extraction and reconstitution of extracellular matrix components involved in local bone differentiation. Proc Natl Acad Sci U S A. 1981;78(12):7599-603.
14. Reddi AH. Morphogenesis and tissue engineering of bone and cartilage: inductive signals, stem cells, and biomimetic biomaterials. Tissue Eng. 2000; 6(4):351-9.

15. Ripamonti U. Soluble osteogenic molecular signals and the induction of bone formation. Biomaterials. 2006;27(6):807-22.

16. Klar RM, Duarte R, Dix-Peek T, Dickens C, Ferretti C, Ripamonti U. Calcium ions and osteoclastogenesis initiate the induction of bone formation by coral-derived macroporous constructs. J Cell Mol Med. 2013;17(11):1444-57.

17. Klar RM, Duarte R, Dix-Peek T, Ripamonti U. The induction of bone formation by the recombinant human transforming growth factor-beta3. Biomaterials. 2014;35(9):2773-88.

18. Ripamonti U, Klar RM, Parak R, Dickens C, Dix-Peek T, Duarte R. Tissue segregation restores the induction of bone formation by the mammalian transforming growth factor-beta(3) in calvarial defects of the non-human primate Papio ursinus. Biomaterials. 2016;86:21-32.

19. Ho SS, Keown AT, Addison B, Leach JK. Cell migration and bone formation from Mesenchymal stem cell spheroids in alginate hydrogels are regulated by adhesive ligand density. Biomacromolecules. 2017;18(12):4331-40.

20. Trueta J. The role of the vessels in osteogenesis. Bone Joint Surg. 1963:45(B): 402-18.

21. Nakagawa H, Takagi K, Kitaoka M, Iyama Kl, Usuku G. Influence of monocytemacrophage lineage cells on alkaline phosphatase activity of developing osteoblasts derived from rat bone marrow stromal cells. Nihon Seikeigeka Gakkai Zasshi. 1993;67(5):480-9.

22. Nguyen BN, Ko H, Fisher JP. Tunable osteogenic differentiation of hMPCs in tubular perfusion system bioreactor. Biotechnol Bioeng. 2016; 113(8):1805-13.

23. Bhumiratana S, Bernhard JC, Alfi DM, Yeager K, Eton RE, Bova J, et al. Tissueengineered autologous grafts for facial bone reconstruction. Sci Transl Med. 2016:8(343):343ra83.

24. Mitra D, Whitehead J, Yasui OW, Leach JK. Bioreactor culture duration of engineered constructs influences bone formation by mesenchymal stem cells. Biomaterials. 2017;146:29-39.

25. Gilbert SF. Developmental biology - Osteogenesis: the development of bones 6th edition. 6th ed. Sunderland, MA: Sinauer Associates; 2000.

26. Nelson DL, Cox M. Lehninger principles of biochemistry. 4th ed. New York: W.H. Freeman and Company; 2005.

27. Blair HC, Larrouture OC, Li Y, Lin H, Beer-Stoltz D, Liu L, et al. Osteoblast differentiation and bone matrix formation in vivo and in vitro. Tissue Eng Part B Rev. 2017;23(3):268-80.

28. Leighton J, Mark R, Justh G. Patterns of three-dimensional growth in vitro in collagen-coated cellulose sponge: carcinomas and embryonic tissues. Cancer Res. 1968;28(2):286-96.

29. Roach HI. Long-term organ culture of embryonic chick femora: a system for investigating bone and cartilage formation at an intermediate level of organization. J Bone Miner Res. 1990;5(1):85-100.

30. Sakakura Y, Fujiwara N, Nawa T. A simple, disposable, and improved organ culture system for maintaining three-dimensional development of mouse embryonic molars. In Vitro Cell Dev Biol. 1989;25(10):959-64.

31. Perniconi B, Coletti D. Skeletal muscle tissue engineering: best bet or black beast? Front Physiol. 2014:5:255.

32. Betz OB, Betz VM, Schroder C, Penzkofer R, Gottlinger M, Mayer-Wagner S, et al. Repair of large segmental bone defects: BMP-2 gene activated muscle grafts vs. autologous bone grafting. BMC Biotechnol. 2013;13:65.

33. Ren B, Betz VM, Thirion C, Salomon M, Jansson V, Muller PE, et al. Osteoinduction within BMP-2 transduced muscle tissue fragments with and without a fascia layer: implications for bone tissue engineering. Gene Ther. 2019:26(1-2):16-28

34. Mastrogiacomo $M$, Scaglione $S$, Martinetti $R$, Dolcini L, Beltrame F, Cancedda $R$, et al. Role of scaffold internal structure on in vivo bone formation in macroporous calcium phosphate bioceramics. Biomaterials. 2006:27(17):3230-7.

35. Ripamonti $U$. The morphogenesis of bone in replicas of porous hydroxyapatite obtained from conversion of calcium carbonate exoskeletons of coral. J Bone Joint Surg Am. 1991;73(5):692-703.

36. Kim J, Ma T. Perfusion regulation of hMSC microenvironment and osteogenic differentiation in 3D scaffold. Biotechnol Bioeng. 2012;109(1): 252-61

37. Tsimbouri PM, Childs PG, Pemberton GD, Yang J, Jayawarna V, Orapiriyakul W, et al. Stimulation of 3D osteogenesis by mesenchymal stem cells using a nanovibrational bioreactor. Nat Biomed Eng. 2017;1(9):758-70. 
38. Freeman FE, Schiavi J, Brennan MA, Owens P, Layrolle P, McNamara LM. $\left(^{*}\right.$ mimicking the biochemical and mechanical extracellular environment of the Endochondral ossification process to enhance the in vitro mineralization potential of human Mesenchymal stem cells. Tissue Eng Part A. 2017;23(2324):1466-78.

39. Bouet G, Cruel M, Laurent C, Vico L, Malaval L, Marchat D. Validation of an in vitro 3D bone culture model with perfused and mechanically stressed ceramic scaffold. Eur Cell Mater. 2015;29:250-66 discussion 66-7.

40. Liu Y, Teoh SH, Chong MS, Yeow CH, Kamm RD, Choolani M, et al. Contrasting effects of vasculogenic induction upon biaxial bioreactor stimulation of mesenchymal stem cells and endothelial progenitor cells cocultures in three-dimensional scaffolds under in vitro and in vivo paradigms for vascularized bone tissue engineering. Tissue Eng Part A. 2013; 19(7-8):893-904

41. Klar RM. The induction of bone formation: the translation enigma. Front Bioeng Biotechnol. 2018;6:74

42. Urist MR, Silverman BF, Buring K, Dubuc FL, Rosenberg JM. The bone induction principle. Clin Orthop Relat Res. 1967;53:243-83.

43. Beier JP, Horch RE, Hess A, Arkudas A, Heinrich J, Loew J, et al. Axial vascularization of a large volume calcium phosphate ceramic bone substitute in the sheep AV loop model. J Tissue Eng Regen Med. 2010;4(3): 216-23.

44. Polykandriotis E, Arkudas A, Beier JP, Hess A, Greil P, Papadopoulos T, et al Intrinsic axial vascularization of an osteoconductive bone matrix by means of an arteriovenous vascular bundle. Plast Reconstr Surg. 2007;120(4):85568

45. Griffith CK, Miller C, Sainson RC, Calvert JW, Jeon NL, Hughes CC, George SC. Diffusion limits of an in vitro thick prevascularized tissue. Tissue Eng. 2005; 11(1-2):257-66.

46. Mayer $H$, Bertram $H$, Lindenmaier W, Korff $T$, Weber $H$, Weich $H$. Vascular endothelial growth factor (VEGF-A) expression in human mesenchymal stem cells: autocrine and paracrine role on osteoblastic and endothelial differentiation. J Cell Biochem. 2005;95(4):827-39.

47. Urist MR. Bone: formation by autoinduction. Science. 1965;150(3698):893-9.

48. Fu H, Rahaman MN, Brown RF, Day DE. Evaluation of bone regeneration in implants composed of hollow HA microspheres loaded with transforming growth factor beta1 in a rat calvarial defect model. Acta Biomater. 2013;9(3): 5718-27.

49. Cassim S, Raymond VA, Lapierre P, Bilodeau M. From in vivo to in vitro: major metabolic alterations take place in hepatocytes during and following isolation. PLoS One. 2017;12(12):e0190366.

50. Bonab MM, Alimoghaddam K, Talebian F, Ghaffari SH, Ghavamzadeh A, Nikbin B. Aging of mesenchymal stem cell in vitro. BMC Cell Biol. 2006;7:14.

51. McKee C, Chaudhry GR. Advances and challenges in stem cell culture. Colloids Surf B Biointerfaces. 2017;159:62-77.

52. Bancroft JD, Gamble M, editors. Theory and practice of histological techniques. London UK: Elsevier health sciences; 2008.

53. Feldman AT, Wolfe D. Tissue processing and hematoxylin and eosin staining. Methods Mol Biol. 2014;1180:31-43.

54. Movat $\mathrm{HZ}$. Demonstration of all connective tissue elements in a single section; pentachrome stains. AMA Arch Pathol. 1955;60(3):289-95.

\section{Publisher's Note}

Springer Nature remains neutral with regard to jurisdictional claims in published maps and institutional affiliations.

Ready to submit your research? Choose BMC and benefit from:

- fast, convenient online submission

- thorough peer review by experienced researchers in your field

- rapid publication on acceptance

- support for research data, including large and complex data types

- gold Open Access which fosters wider collaboration and increased citations

- maximum visibility for your research: over $100 \mathrm{M}$ website views per year

At BMC, research is always in progress.

Learn more biomedcentral.com/submissions 